\title{
Structure-orientated fluorescence photobleaching analysis: a method for double fluorescent labelling studies ${ }^{1}$
}

\author{
R. I. GHAUHARALI, ${ }^{*}$ R. VAN DRIEL $†$ \& G. J. BRAKENHOFF* \\ * BioCentrum Amsterdam, Institute for Molecular Cell Biology, Kruislaan 316, \\ 1098 SM Amsterdam, The Netherlands \\ $\dagger$ E.C. Slater Instituut, University of Amsterdam, Plantage Muidergracht 12, \\ 1018 TV Amsterdam, The Netherlands
}

Key words. Fluorescence photobleaching, fluorescence fading, double fluorescent labelling, confocal microscopy, CCD camera.

\begin{abstract}
Summary
Differences in the degree of photodegradation can be used for fluorophore identification in double fluorescently labelled specimens. Based on the use of morphological information, a noise-insensitive method is presented for discriminating between the fluorophores, assuming spatially uniform photodegradation. Separate images of the labelled structures can be obtained. Alternatively, with spatially nonuniform photodegradation, the photodynamics of one fluorophore - i.e. photodegradation, concentration associated quenching, etc. - in relation to its microenvironment can be investigated.
\end{abstract}

\section{Introduction}

Fluorescence microscopy is one of the most powerful techniques for localizing and studying specific components in cells and tissues. Often it is important to analyse the distribution of several components simultaneously. Discrimination between the fluorophores used to label these components is usually based on the spectral characteristics of these fluorophores. By carefully selecting excitation and emission filter combinations, it is possible to detect the signal of one fluorophore, whilst other signals are largely suppressed. It has been shown that the emissions from at least five different fluorophores can be distinguished in the same cell using such an approach (DeBasio et al., 1987). A complication in these experiments is that when fluorophores are used with overlappping excitation and/or emission spectra, discrimination becomes difficult, which leads to cross-talk between the fluorophores. Several methods have been developed to reduce this cross-talk. These are based on

\footnotetext{
${ }^{1}$ Presented at 3D Imaging Sciences in Microscopy, Oxford, 14-17 April 1996. Correspondence to: G. J. Brakenhoff. Tel: +31-20-5255189; fax: +31-205256271; e-mail: brakenhoff@mc.bio.uva.nl.

(C) 1997 The Royal Microscopical Society
}

electronic (Loken et al., 1977) or mathematical (Carlsson \& Mossberg, 1992) compensation of the detected signal for the error signal. In another approach, Carlsson et al. (1994) introduced a method in which two excitation beams are intensity modulated, each with a specific modulation frequency. Discrimination between the fluorophores was based on spectral fluorophore characteristics and the excitation intensity modulation frequency.

In this report we introduce a new method to carry out double fluorescent labelling studies. Following earlier work on bleach rate imaging (Brakenhoff et al., 1994), the proposed method employs differences in photostability rather than spectral differences to discriminate between the fluorophores used. In this method, two images of the specimen are acquired, using a (bilateral) confocal scanning laser microscope and cooled charge-coupled device (CCD) camera detection (Brakenhoff \& Visscher, 1992, 1993). Following the first image, a second image is acquired after the specimen is partially bleached. These images are analysed by comparing the fluorescence intensity of structures as a whole - as opposed to single pixels, done earlier by Brakenhoff et al. (1994) - in the images before and after bleaching. From such a comparison, the degree of photodegradation of the two fluorophores can be determined and with this information, images of the separate distribution of the two fluorophores can be calculated, provided that bleaching of the fluorophores is spatially uniform. When bleaching is spatially nonuniform, information about the microenvironment for one specific fluorophore can be obtained.

Any small image shifts that occur during the bleaching process, caused by, for example, mechanical instabilities of the microscope, would lead to incorrect local estimates for the degree of photodegradation. It is therefore essential that the images are aligned correctly before they are compared. This is achieved by mathematically aligning the images 
after acquisition, using a method based on Fourier and correlation techniques with subpixel resolution.

\section{Theoretical description}

\subsection{Principle of the method}

In the proposed approach to double fluorescent labelling studies, identification of the fluorophores is based on their relative photostability. Ideally, the selected fluorophores should have significantly different photostabilities. From a specimen labelled with such fluorophores, two images are obtained, separated by a time interval $\Delta t$ over which the specimen is bleached to a certain degree. We will refer to the images before and after bleaching as $I_{1}(\boldsymbol{r})$ and $I_{2}(\boldsymbol{r})$, respectively; the vector $r$ denotes a position within the image. From these two images, the individual distribution of the fluorophores in the specimen can be determined, provided that the fluorophores label distinct morphologically identifiable structures. This can be done as follows. For a specimen labelled with two fluorophores, $A$ and $B$, the intensities in the images before and after bleaching, $I_{1}(\boldsymbol{r})$ and $I_{2}(\boldsymbol{r})$, can be written as

$$
\begin{aligned}
& I_{1}(\boldsymbol{r})=F_{A}(\boldsymbol{r})+F_{B}(\boldsymbol{r}) \\
& I_{2}(\boldsymbol{r})=x_{A} F_{A}(\boldsymbol{r})+x_{B} F_{B}(\boldsymbol{r})
\end{aligned}
$$

in which $F_{A}(\boldsymbol{r})$ and $F_{B}(\boldsymbol{r})$ represent the distribution of fluorescence intensity of the fluorophores $A$ and $B$ in the specimen. $x_{A}$ and $x_{B}\left(x_{A}, x_{B} \leqslant 1\right)$ are so-called bleach factors, which indicate the degree of photodegradation during the time interval $\Delta t$. These bleach factors can be determined from the images $I_{1}(\boldsymbol{r})$ and $I_{2}(\boldsymbol{r})$ as follows. To determine the bleach factor of fluorophore $B$ we define an adjustable constant $\varepsilon_{B}$ and subtract the image after bleaching, $I_{2}(\boldsymbol{r})$, multiplied by this constant, from the image before bleaching, $I_{1}(\boldsymbol{r})$ :

$$
I_{1}(\boldsymbol{r})-\varepsilon_{B} I_{2}(\boldsymbol{r})=F_{A}(\boldsymbol{r})\left(1-\varepsilon_{B} x_{A}\right)+F_{B}(\boldsymbol{r})\left(1-\varepsilon_{B} x_{B}\right) .
$$

If the constant $\varepsilon_{B}$ is chosen in such a way that, after subtraction, structures labelled with the fluorophore $B$ can no longer be distinguished from the background, i.e.

$$
\left(1-\varepsilon_{B} x_{B}\right)=0
$$

the bleach factor can be directly related to the adjustable constant:

$$
x_{B}=\frac{1}{\varepsilon_{B}} .
$$

This procedure is illustrated in Fig. 1 using a computergenerated object consisting of two disc-like structures labelled with a photo-unstable $(B)$ and a photostable $(A)$ model fluorophore, respectively. Figure 1(a) shows the image before bleaching, $I_{1}(\boldsymbol{r})$. In Fig. 1(b) the image after bleaching, $I_{2}(\boldsymbol{r})$, which is calculated using Eq. (1) and the arbitrarily chosen bleach factors $x_{A}=0.9$ and $x_{B}=0.5$, is shown. The background in this image was chosen to bleach with a bleach factor of $x_{\text {back }}=0.95$. In Figs. $1(\mathrm{c})-1(\mathrm{e})$, the adjustable constant $\varepsilon$ for structure $B$ is determined. In the images $1(\mathrm{c})$ and $1(\mathrm{e})$, the structure labelled with fluorophore $B$ is still visible, while in image $1(d)$, the structure can no longer be distinguished from the background. The optimum value for $\varepsilon_{B}=2 \cdot 0$, found from image $1(\mathrm{~d})$, indeed agrees with that expected from Eq. (4). The accuracy with which the optimum value can be determined depends on the signal-to-noise ratio in the image. The bleach factor of fluorophore $A$ is determined in a similar way, with the help of an adjustable constant $\varepsilon_{A}$. It is found that

$$
x_{A}=\frac{1}{\varepsilon_{A}} .
$$

For the example shown in Fig. 1, structure $A$ could not be distinguished from the background for $\varepsilon_{A}=1 \cdot 1$ (images not shown), which corresponds well with the bleach factor $x_{A}=0 \cdot 9$, as calculated with Eq. (5).

The distribution of the fluorophores $A$ and $B, F_{A}(\boldsymbol{r})$ and $F_{B}(\boldsymbol{r})$, can be determined from the images before and after bleaching with the help of the bleach factors $x_{A}$ and $x_{B}$. Substitution of Eqs. (3)-(5) in Eq. (2) gives the separate images of the fluorophores $A$ and $B$ :

$$
\begin{aligned}
& F_{A}(\boldsymbol{r})=\frac{I_{1}(\boldsymbol{r})-I_{2}(\boldsymbol{r}) / x_{B}}{1-x_{A} / x_{B}} \\
& F_{B}(\boldsymbol{r})=\frac{I_{1}(\boldsymbol{r})-I_{2}(\boldsymbol{r}) / x_{A}}{1-x_{B} / x_{A}} .
\end{aligned}
$$

In Figs. 1(f) and 1(g), the calculated distribution of the two fluorophores is shown using Eqs. (4)-(7) and the values for $\varepsilon_{A}$ and $\varepsilon_{B}$, found from the analysis illustrated in Figs. 1(c)$1(\mathrm{e}): \varepsilon_{A}=1 \cdot 1$ and $\varepsilon_{B}=2 \cdot 0$. These images show that it is possible to obtain images specific for one fluorophore and that the signal of the other fluorophore can be completely suppressed. Furthermore, the determination of the bleach factors could be done in the presence of a bleaching background. In general, the analysis presented here is possible when the structures have a common background; whether this background is bleaching or not is of no importance.

It is important to point out that the method for determining the adjustable constants $\varepsilon$ - and thus the distribution of the fluorophores in the specimen - depends on the comparison of intensities in the images before and after bleaching, combined with morphological information about the structures in the specimen. Since the optimum value for $\varepsilon$ is reached when the entire structure is indistinguishable from the background, all pixels which make up the structure are analysed simultaneously. This aspect of the analysis makes the present method less susceptible to pixel-to-pixel intensity variations than the earlier methods based on a single pixel approach.

Note that it is assumed in the analysis that the bleach factors are constant throughout the specimen. If this condition is not fulfilled, it is not possible with this method 

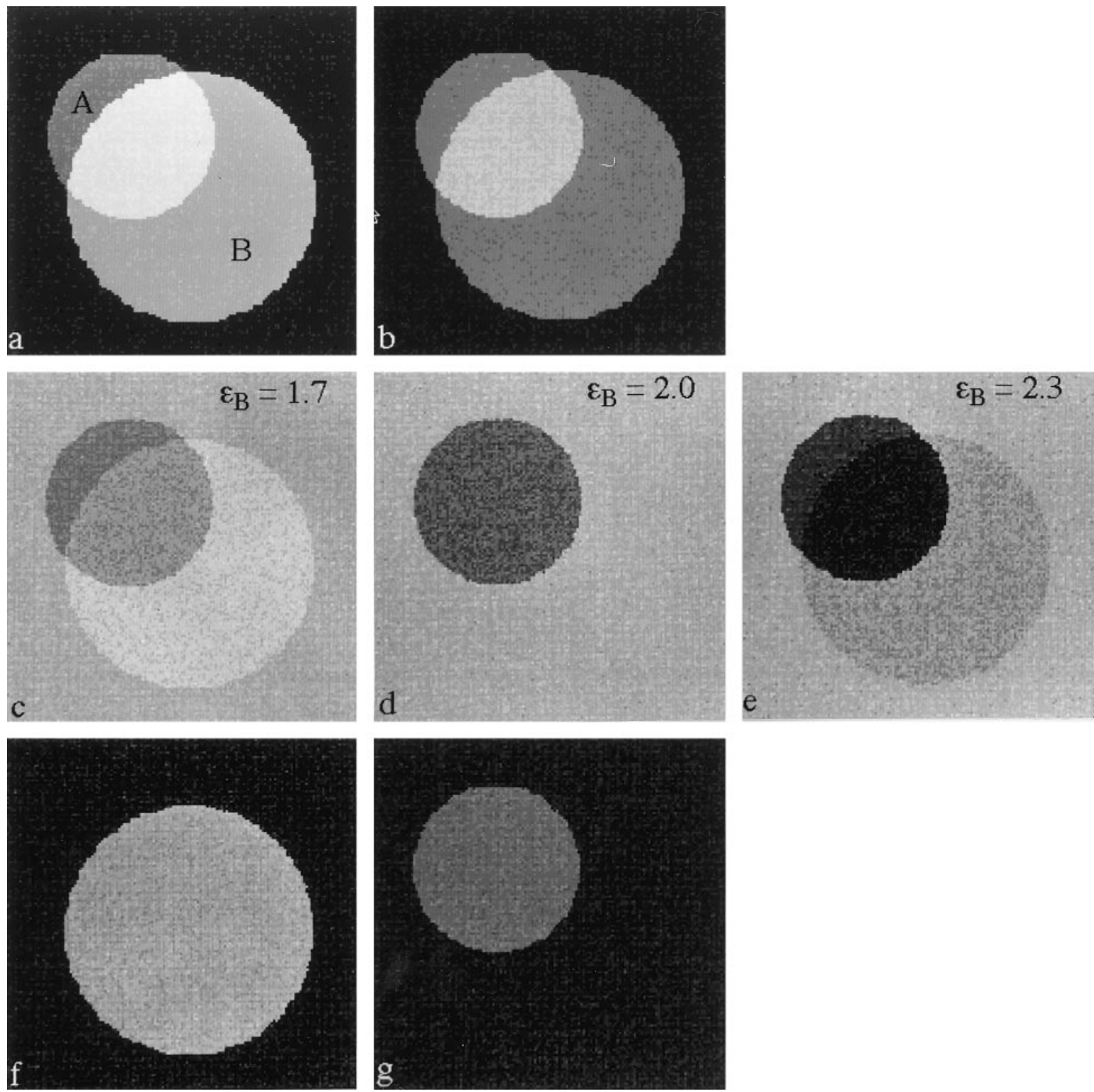

Fig. 1. Schematic illustration of the analysis. (a) Computer-generated model object before bleaching consisting of two structures, each labelled with one fluorophore $(A$ and $B$ ). The shape and number of structures was chosen arbitrarily. Gaussian noise - the same amount for each intensity level - was added. (b) Model object after bleaching, calculated from image (a) (see text for details). (c)-(e) Determination of the adjustable constant for fluorophore $B, \varepsilon_{B}$. The images shown were calculated by subtracting the image after bleaching, multiplied by $\varepsilon_{B}$, from the image before bleaching. The three images correspond to three values of $\varepsilon_{B}$. For $\varepsilon_{B}=2 \cdot 0$ (image (d)) the structure labelled with fluorophore $B$ cannot be distinguished from the background. This image determines the optimum value for $\varepsilon_{B}$. (f) and (g) Reconstruction of the separate distribution of the fluorophores $A$ and $B$. These images were calculated from the images before and after bleaching, using the optimum values for the adjustable constants $\varepsilon_{A}, \varepsilon_{B}$ and Eqs. (4)-(7).

to calculate images of the separate distribution of the two fluorophores. When photodegradation is spatially nonuniform, structure-orientated photobleaching analysis can be used to investigate the factors determining the observed variation in photodegradation behaviour for one specific fluorophore.

\subsection{Image alignment}

A prerequisite for the correct determination of the adjustable constants $\varepsilon$ is that the images before and after bleaching, $I_{1}(\boldsymbol{r})$ and $I_{2}(\boldsymbol{r})$, are aligned correctly. However, small image shifts can occur during the time over which bleaching is accomplished, for example due to drifting of the stage of the microscope. To correct for these image shifts, the images are mathematically realigned after acquisition. The alignment is carried out using Fourier techniques, which allows images to be translated over subpixel ranges. According to the Fourier shift theorem (Bracewell, 1965; Larkin et al., 1991), a translation in the spatial domain corresponds to a linear phase shift in the spatial frequency domain. This means that an image can be translated by an 

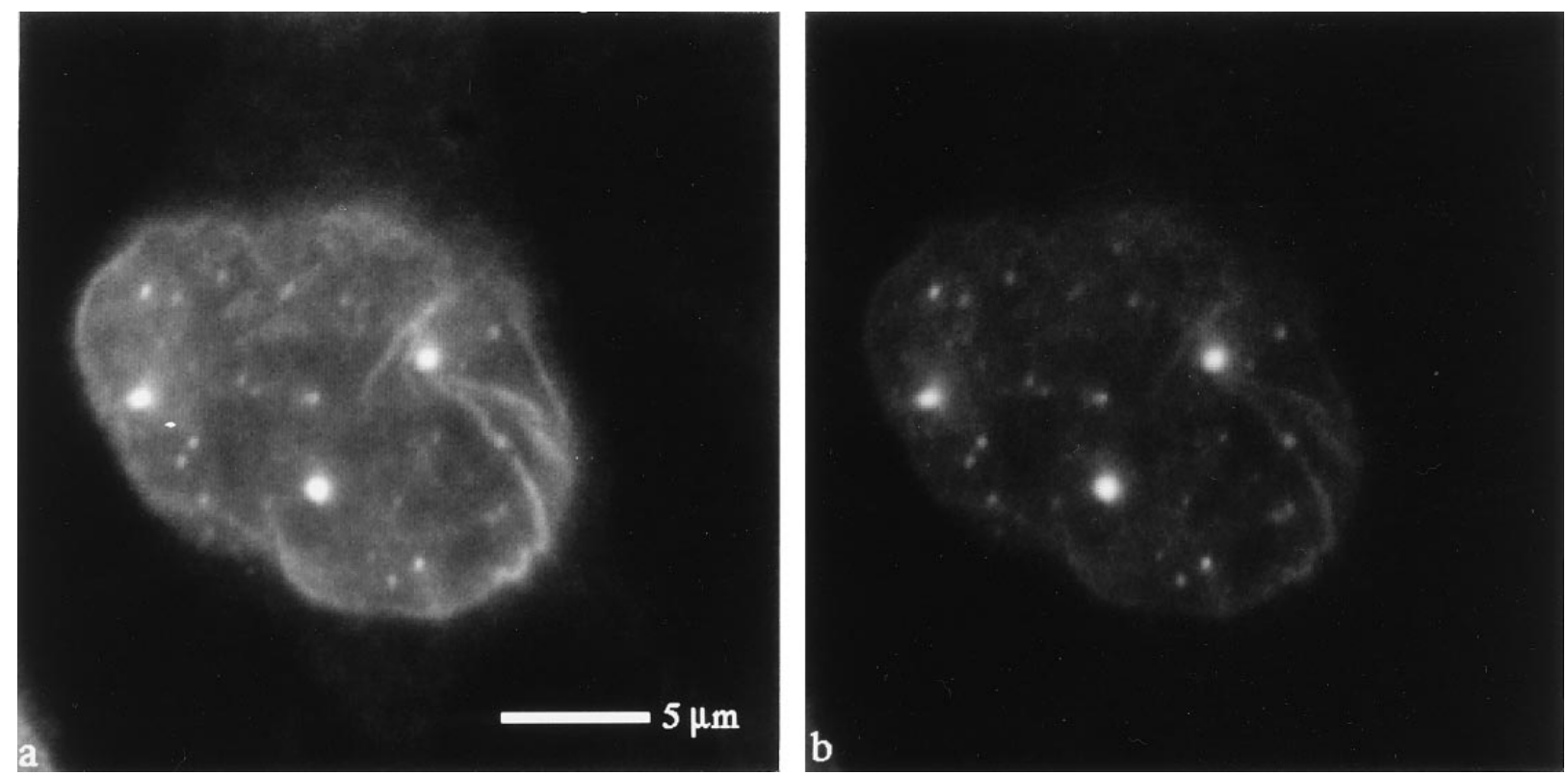

Fig. 2. Images of a nucleus of a HeLa cell in which the nuclear envelope and certain nuclear domains, coiled bodies, are fluorescently labelled with the fluorophores FITC and $\mathrm{Cy} 3$, respectively: (a) before bleaching; (b) after bleaching.

appropriate phase transformation of its Fourier transform. The phase transformation needed is a phase addition, where the magnitude of the addition at a certain spatial frequency is proportional to that frequency. For a translation over a range of one pixel in a particular direction, the proportionality constant is equal to $\pi$ over the highest spatial frequency in that direction. Translations over ranges smaller than one pixel are accomplished by linearly varying the proportionality constant. The translation needed for correct alignment of the two images is determined by finding the maximum of the cross-correlation function between these images. This is done in two steps. First, the cross-correlation of the images $I_{1}(\boldsymbol{r})$ and $I_{2}(\boldsymbol{r})$ is calculated for a number of translations of the image $I_{2}(\boldsymbol{r})$. Second, these calculated cross-correlation values are approximated locally by a parabolic surface. The maximum of this surface is determined with a bracketing and bisection method (Press et al., 1992). This maximum corresponds to the translation needed for correct alignment of the two images.

\section{Experiments}

\subsection{Experimental set-up and specimen preparation}

Image acquisition was with an Olympus IMT-2 inverted microscopy (Olympus Corporation, Lake Success, NY, U.S.A.), equipped with an INSIGHT PLUS bilateral confocal line scanning unit (Meridian Instruments Inc., Okemos, MI, U.S.A.) (Brakenhoff \& Visscher, 1992, 1993) and an oilimmersion objective lens $(100 \times, \mathrm{NA}=1 \cdot 32)$. An air-cooled argon ion laser (model 532, Omnichrome, Chino, CA, U.S.A.) was used for fluorescence excitation at $488 \mathrm{~nm}$. Fluorescence detection as with a cooled CCD camera (model DDE/3200, Astromed, Cambridge, U.K.). A Hewlett-Packard model 725/50 workstation (Hewlett-Packard, Palo Alto, CA, U.S.A.) was used for image collection and exposure control via a mechanical shutter. The image exposure time and the bleach time interval were chosen to be 30 and $150 \mathrm{~s}$, respectively. These values yielded images with a good signal-to-noise ratio and sufficient bleaching to determine the degree of photodegradation. Image analysis was carried out on the same workstation, using the image processing package Scillmage (Ten Kate et al., 1990), supplemented with custom-made routines for the determination of the adjustable constant $\varepsilon$ and the optimal translation for correct image alignment.

HeLa cells (human cervix carcinoma) were used for indirect immunofluorescent labelling. With these cells, two specimens, mounted in glycerol, were prepared. In specimen A, the nuclear envelope was fluorescently labelled with the antibody/fluorophore combination 41CC4/FITC. Certain domains in the nucleus, referred to as 'coiled bodies' were labelled with the antibody $\alpha \mathrm{P} 80$ combined with the fluorophore Cy3 (Carmo-Fonseca et al., 1993). In specimen $\mathrm{B}$, two types of nuclear domain were labelled. The antibody $5 \mathrm{E} 10$, combined with the fluorophore $\mathrm{Cy} 3$, was used to label nuclear domains referred to as 'PML bodies' (Stuurman et al., 1992; Grande et al., 1996) and the antibody/ fluorophore combination $\alpha$ P80/FITC to visualize coiled bodies. For culture and labelling procedures, see Schul et al. (1996). 


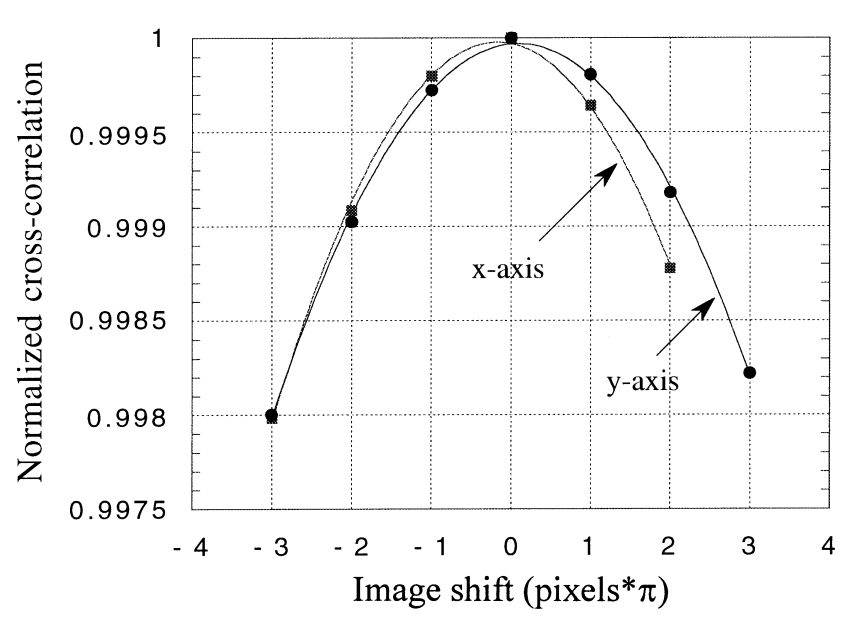

Fig. 3. Verification of the alignment of the images before and after bleaching shown in Fig. 2. The relative cross-correlation of the image before and after bleaching as a function of the translation of the image after bleaching along the horizontal $(x)$ and the vertical $(y)$ axis is plotted. The solid lines represent fits with a second-order polynomial. The maximum cross-correlation is found for translations of less than $0 \cdot 1$ pixels in either direction.

\subsection{Image decomposition}

In Fig. 2 a set of typical images of specimen A before and after bleaching are shown. The nuclear envelope and coiled bodies in the nucleus of a HeLa cell, fluorescently labelled

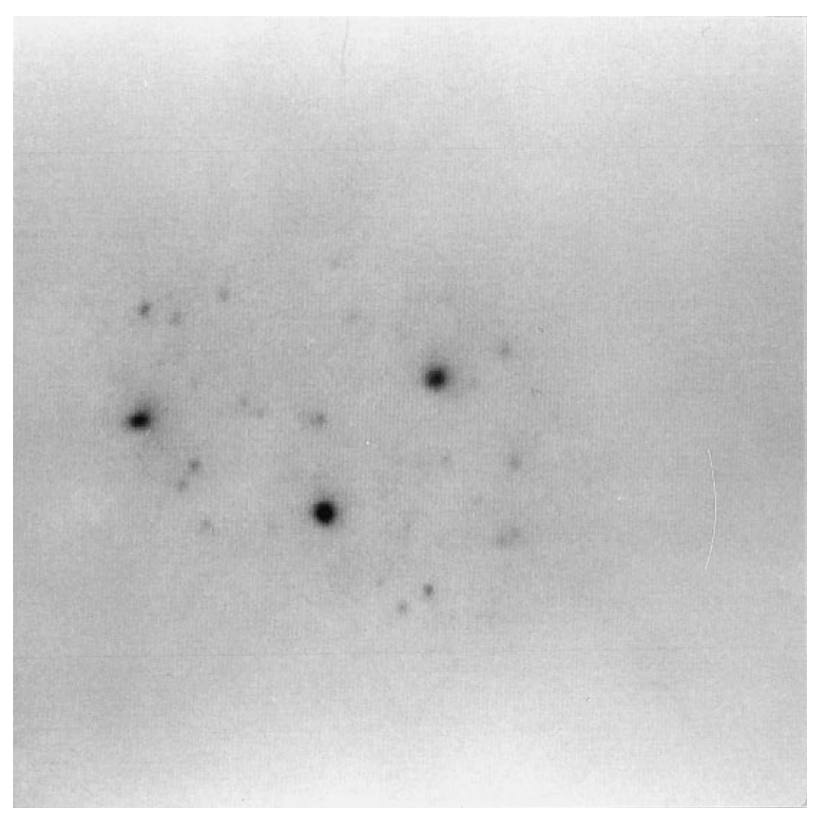

Fig. 4. Determination of the adjustable constant for the fluorophore labelling the nuclear envelope, FITC. The image $I_{1}(\boldsymbol{r})-\varepsilon_{\mathrm{FITC}} I_{2}(\boldsymbol{r})$ for the optimum value $\varepsilon_{\text {FITC }}=2.5$ is shown. Only the coiled bodies are visible and the image contribution of the nuclear envelope has disappeared. The properties of the background matching procedure for the nuclear envelope caused inversion of the apparent contrast. with FITC and $\mathrm{Cy} 3$ respectively, are visible. In the image after bleaching - Fig. 2(b) - the fluorescence intensity of the nuclear envelope is significantly lower than before bleaching, while that of the coiled bodies has decreased only marginally. This indicates a lower photostability of the FITClabelled structures than those labelled with $\mathrm{Cy} 3$ under the present circumstances.

The first step in the analysis is the correction of possible misalignment of the images before and after bleaching, $I_{1}(\boldsymbol{r})$ and $I_{2}(\boldsymbol{r})$. As described, this is done by mathematically translating the image $I_{2}(\boldsymbol{r})$ and calculating, for each translation, the cross-correlation of the first and translated second image. The maximum cross-correlation corresponds to the translation of the image $I_{2}(\boldsymbol{r})$ needed for correct alignment. In Fig. 3, the normalized cross-correlation is shown for translations of the image after bleaching along the horizontal $(x)$ and vertical $(y)$ axis. For this specific image combination, it was found that a translation of less than $0 \cdot 1$ pixels in both the $x$ - and $y$-direction was needed to attain correct alignment. Since such small translations do not represent significant mechanical displacements, no realignment of the images was necessary and the images in Fig. 2 were used directly in the following analysis.

The second step in the analysis is the determination of the adjustable constants $\varepsilon_{\mathrm{FITC}}$ and $\varepsilon_{\mathrm{Cy} 3}$. As described, this is done by calculating the image $I_{1}(\boldsymbol{r})-\varepsilon I_{2}(\boldsymbol{r})$ for a number of values of $\varepsilon$ and determining for which value of $\varepsilon$ the structure of interest can no longer be distinguished from the background. In Fig. 4 the adjustable constant for the nuclear envelope is estimated. The image $I_{1}(\boldsymbol{r})-\varepsilon I_{2}(\boldsymbol{r})$ for $\varepsilon=2.5$ is shown. For this value, only the coiled bodies are visible and the nuclear envelope has disappeared. The uncertainty in this value is estimated to be $0 \cdot 1$. Thus it is found that the adjustable constant for the fluorophore labelling the nuclear envelope, FITC, is: $\varepsilon_{\text {FITC }}=2 \cdot 5 \pm 0 \cdot 1$. The adjustable constant for $\mathrm{Cy} 3$ is determined in a similar way. It was found that: $\varepsilon_{\mathrm{Cy} 3}=1 \cdot 1 \pm 0 \cdot 1$. The corresponding bleach factors, $x_{\mathrm{FITC}}$ and $x_{\mathrm{Cy} 3}$, are determined using Eqs. (4) and (5): $x_{\mathrm{FITC}}=0.4$ and $x_{\mathrm{Cy} 3}=0.9$. The separate distributions of FITC and $\mathrm{Cy} 3$ can now be calculated with these bleach factors and Eqs. (6) and (7). The results of this calculation are shown in Fig. 5. As can be seen, nearly complete separation of the nuclear envelope and the coiled bodies has been obtained. In the FITC-labelled nuclear envelope image (Fig. 5(a)), elongated white regions (indicated by the arrows) clearly show up, possible representing folds in the nuclear membrane. In Fig. 5(b) an enhanced visibility of the Cy3-labelled coiled bodies results, with finer elements becoming apparent.

\subsection{Spatially nonuniform photodegradation}

A second application of structure-orientated photodegradation imaging is the analysis of the spatial dependence of 

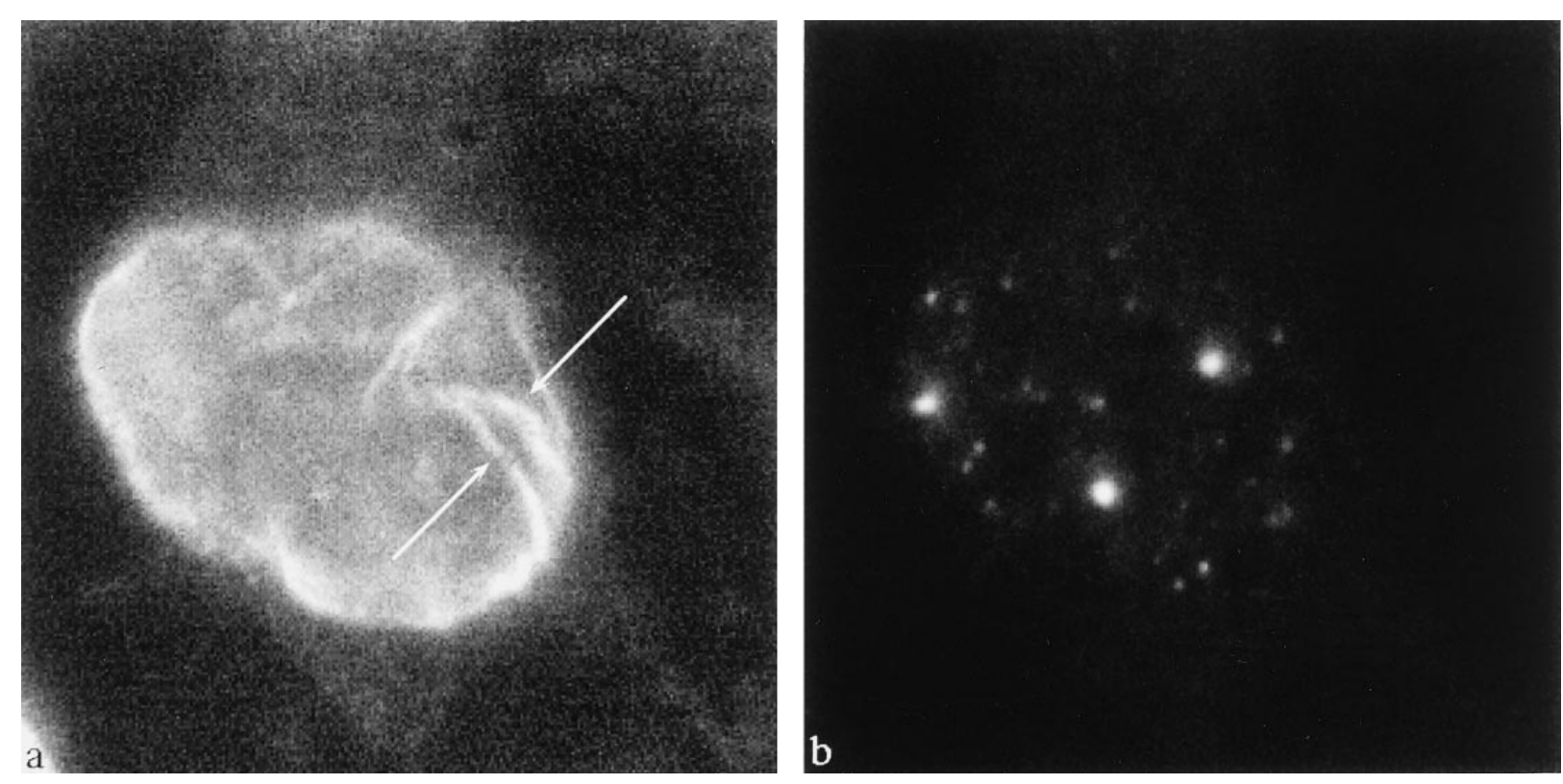

Fig. 5. Determination of the separate distribution of the fluorophores FITC and Cy3. These images were calculated using Eqs. (4)-(7) and the optimum values for $\varepsilon_{\mathrm{FITC}}$ and $\varepsilon_{\mathrm{Cy} 3}$. (a) Image of the FITC-labelling. In this image, the signal of Cy3 is absent. (b) Image of the Cy3labelling. In this image, no signal of FITC is present. Different grey-scales were used for the images, so that the intensities cannot be compared directly.

photodegradation of a specific fluorophore. In the first application - Section 3.2 - separate images of cell structures labelled with different fluorophores were obtained, assuming spatially uniform bleaching, i.e. bleaching which is concentration and environment independent. If this is not the case and it is known that a structure has been labelled with a specific fluorophore, the present method can be used to analyse the spatial variation of photodegradation. The observed differences in the degree of photodegradation may contain valuable information about the concentration and microenvironment of the fluorophore.

To illustrate this application, we show in Fig. 6 a set of

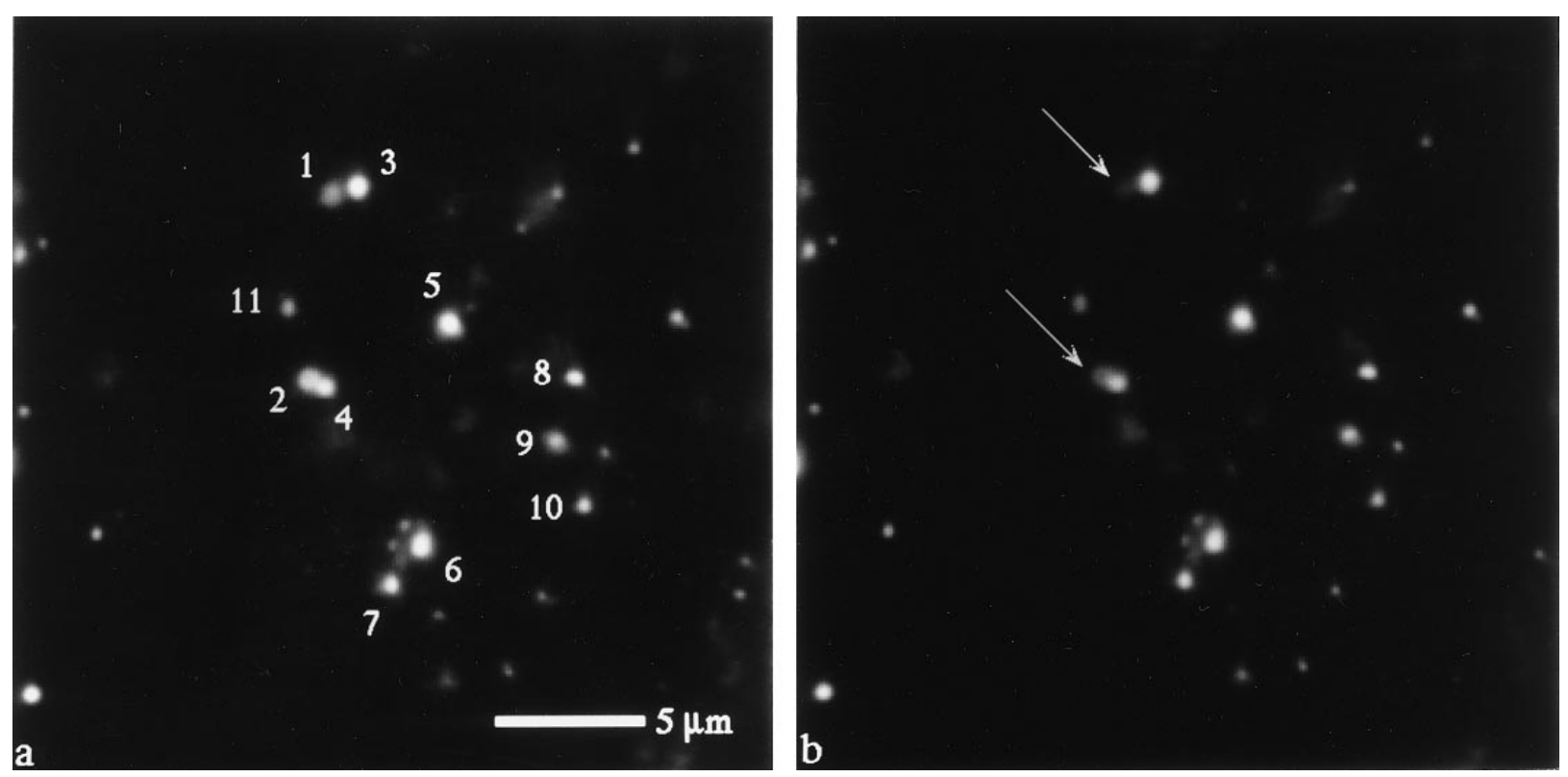

Fig. 6. Images of specimen B representing the nucleus of a HeLa cell in which two types of nuclear domain, PML bodies and coiled bodies, are fluorescently labelled with the fluorophores $\mathrm{Cy} 3$ and FITC, respectively. (a) Before bleaching. Eleven structures which will be used in the analysis are indicated. (b) After bleaching. Two structures with a significantly decreased fluorescence intensity are indicated with arrows. 


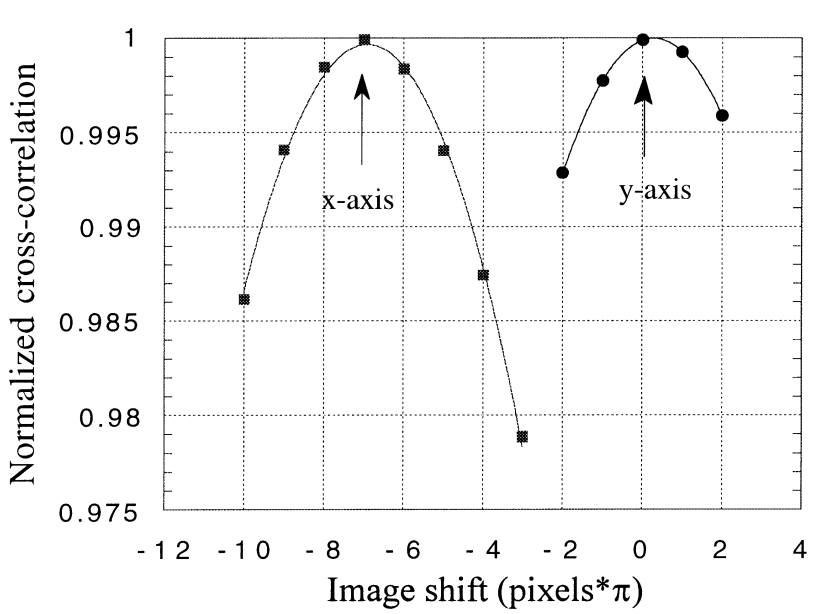

Fig. 7. Verification of the alignment of the images before and after bleaching, shown in Fig. 6. In a similar procedure as illustrated in Fig. 3, it is found that a translation of $2 \cdot 2$ pixels in the negative $x$-direction (i.e. a translation to the left) and $0 \cdot 1$ pixels in the positive $y$-direction (i.e. a translation upwards) of the image after bleaching is needed to attain correct alignment.

typical images of specimen B. In this specimen, two types of domain in the nuclei of HeLa cells, PML bodies and coiled bodies, are fluorescently labelled with $\mathrm{Cy} 3$ and FITC, respectively. After bleaching (Fig. 6(b)), two structures are present, indicated with the arrows, with a significantly decreased fluorescence intensity. Based on the lower photostability of FITC relative to $\mathrm{Cy} 3$, these data indicate that these faster bleaching structures are the FITC-labelled coiled bodies and that the more photostable structures are the Cy3-labelled PML bodies.

The analysis of this specimen proceeds along the same lines as that of specimen A. First, the alignment of the images before and after bleaching, $I_{1}(\boldsymbol{r})$ and $I_{2}(\boldsymbol{r})$, is verified. Using the techniques already described - the results are shown in Fig. 7 - the translation of the image after bleaching required for correct alignment was calculated. In Fig. 8(a) the difference between the image before and after bleaching (prior to misalignment correction) is shown. Inspection of this figure shows a 'shadow' at the right-hand side of each structure in the uncorrected difference image and the absence of any 'shadowing' effects after correct alignment. The lateral shift that occurred in this measurement could be accompanied by a shift in the axial direction. However, we expect that during the bleach time interval, the axial drift is small when compared with the FWHM of the point spread function in this direction - calculated to be approximately $0 \cdot 7 \mu \mathrm{m}$, using the numerical aperture of the objective lens: 1.32 and the excitation wavelength: $488 \mathrm{~nm}$ (Pawley, 1995) - so that drifts in the axial direction will have only a marginal effect.

Second, the adjustable constants for FITC and Cy3 are determined. For FITC (labelling the structures indicated in Fig. 6(b)) it was found that $\varepsilon_{\text {FITC }}=3 \cdot 0 \pm 0 \cdot 1$. Note that this value agrees well with the value of $\varepsilon_{\text {FITC }}=2 \cdot 5 \pm 0 \cdot 1$ for FITC bleaching in specimen A (see Section 3.2), given the
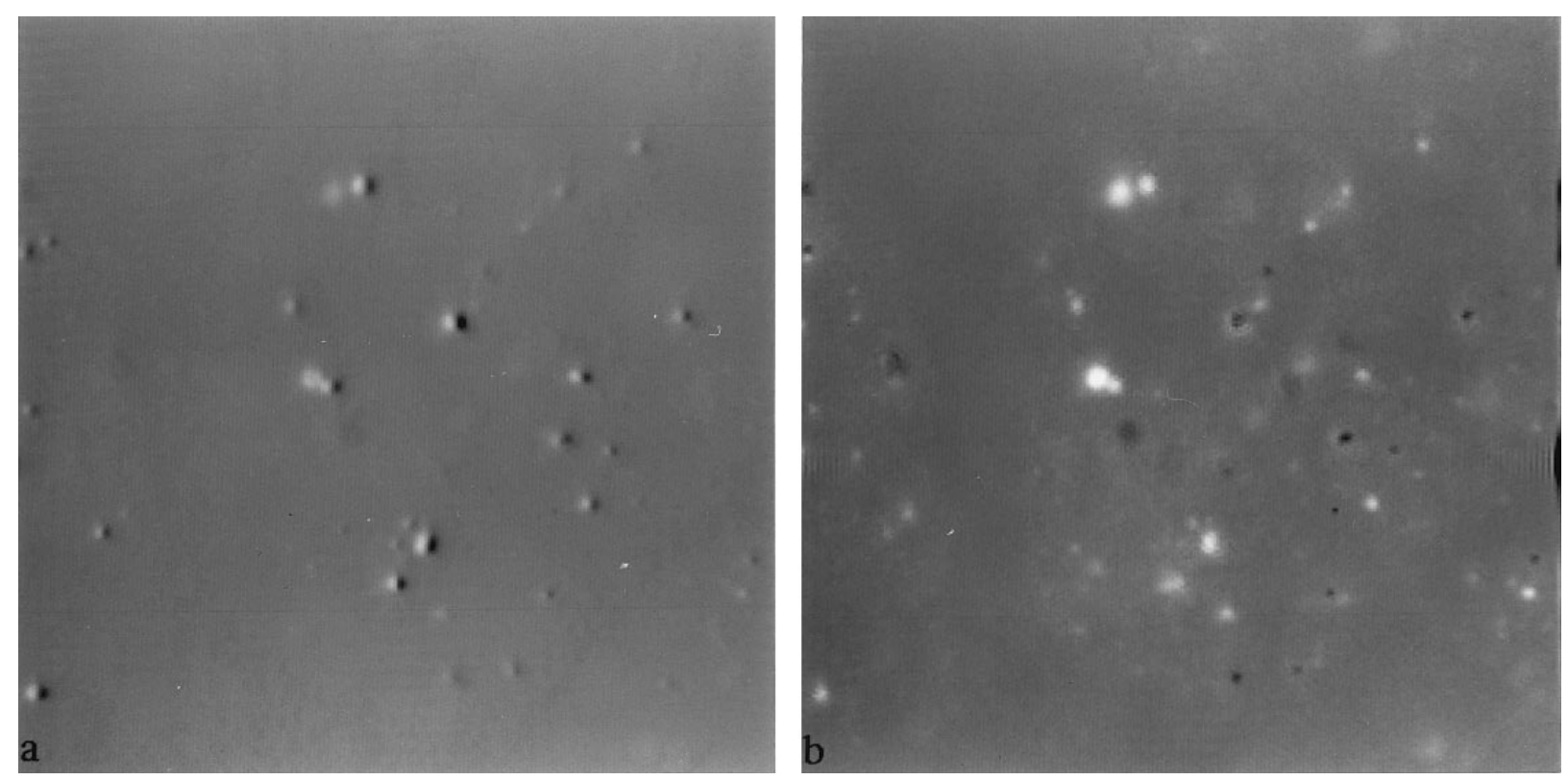

Fig. 8. Images representing the difference between the images before and after bleaching, shown in Fig. 6. (a) Difference between the images before and after bleaching. The 'shadow' at the right-hand side of each structure indicates incorrect alignment of the two images. (b) Difference between the images before and after bleaching when the image after bleaching was mathematically translated over a range of $2 \cdot 2$ pixels in the negative $x$-direction and $0 \cdot 1$ pixels in the positive $y$-direction. The absence of any 'shadowing' effects indicates correct alignment. 
Table 1. Experimentally determined bleach factors for specimen B.

\begin{tabular}{ll}
\hline Nuclear domain & Bleach factor \\
\hline 1 & $0 \cdot 3$ \\
2 & $0 \cdot 4$ \\
3 & $0 \cdot 8$ \\
4 & $0 \cdot 7$ \\
5 & $1 \cdot 1$ \\
6 & $0 \cdot 8$ \\
7 & $0 \cdot 9$ \\
8 & $0 \cdot 9$ \\
9 & $1 \cdot 2$ \\
10 & $0 \cdot 7$ \\
11 & $0 \cdot 7$ \\
\hline
\end{tabular}

day-to-day variations of the output power of the argon ion laser used for fluorescence excitation. Identical exposure times and bleach time intervals were used in the two experiments, carried out a few days apart.

No single value for $\varepsilon_{\mathrm{Cy} 3}$ could be found for which the structures not indicated in Fig. 6(b) disappeared simultaneously, which means that their bleach factor varies from structure to structure. We determined the bleach factor of the structures individually as follows. The images before and after bleaching were divided into small regions of interest, containing only one structure indicated in Fig. 6(a) and the analysis in Eqs. (1)-(5) was carried out on each region of interest separately. The results of this analysis, shown in Table 1, quantify the strong variation in bleach factors. This is probably caused by variations of the microenvironment of the fluorophore (see also the Discussion). The results in this table also show two nuclear bodies with a bleach factor larger than 1 , indicating an increasing fluorescence intensity during exposure to the fluorescence excitation radiation. A possible explanation is that this behaviour is caused by concentration-dequenching. This phenomenon occurs at high fluorophore concentrations, when the fluorescence is quenched as a result of fluorophore-fluorophore interactions. Photodegradation reduces the effective fluorophore concentration, causing the probability for these interactions to decrease and the fluorescence intensity to increase. Also in the corrected difference image, shown in Fig. 8(b), this effect becomes visible as dark spots.

Since the assumption of a constant bleach factor is clearly not satisfied for $\mathrm{Cy} 3$, it is not possible to obtain an image of the FITC labelling in which the signal of $\mathrm{Cy} 3$ is absent. It is however possible to calculate an image of the $\mathrm{Cy} 3$ labelling in which the signal of FITC is suppressed. In this image, shown in Fig. 9, only the Cy3-labelled PML bodies are visible and the FITC-labelled coiled bodies, indicated in Fig. 6(b), are absent.

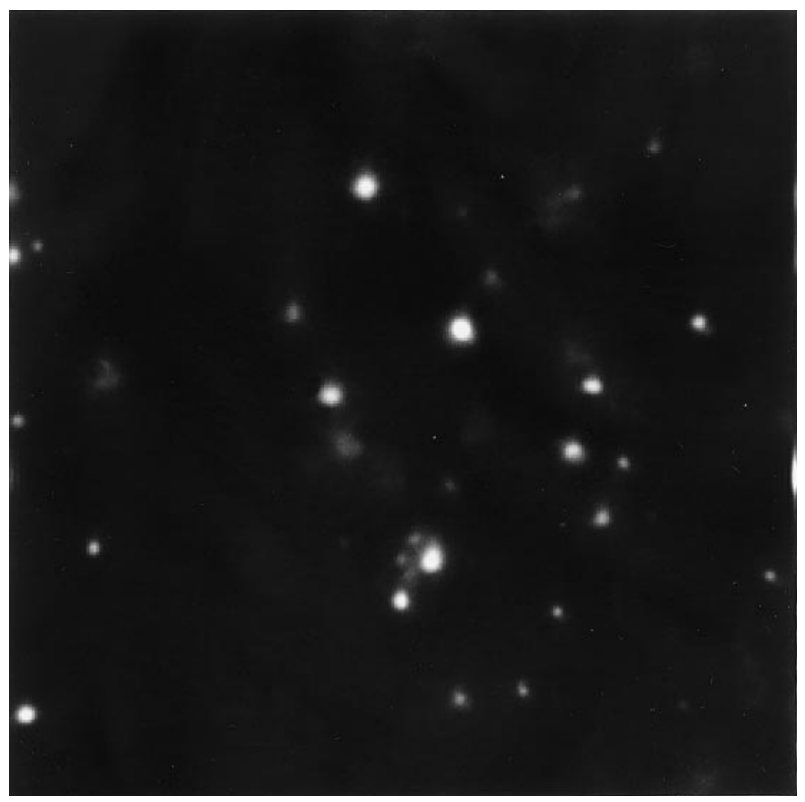

Fig. 9. Separate image of the Cy3-labelled PML bodies. This image was calculated from the images before and after bleaching, the optimum value for the adjustable constant for FITC and mean value for that of $\mathrm{Cy} 3$, as determined from Table 1.

\section{Discussion}

In this paper we present a new approach to double fluorescent labelling studies in which identification of the fluorophores is based on their degree of photodegradation, rather than their spectral properties. Of key importance in the method is that the analysis is based on the comparison of fluorescence intensities of entire structures, rather than single pixels, in the images before and after bleaching. The advantage of this approach is that the analysis is less susceptible to image noise than methods based on single pixel analysis. This problem was encountered in previous work on the use of photodegradation for fluorophore identification (Brakenhoff et al., 1994). In that approach, the bleach factors were determined from so-called bleach maps, which were generated from the images before and after bleaching. The inherent noise in these images could lead to bleach maps which were strongly affected by noise. In those cases, the various bleach factors could not, or only with a limited accuracy, be derived from the bleach maps. The structure-orientated photodegradation analysis presented here is far more robust, permitting relatively sensitive determination of the degrees of photodegradation, even in the presence of noise.

The images in these experiments were acquired using a confocal microscope equipped with a cooled CCD camera for fluorescence detection. Thanks to the large dynamic range and linear response of this camera it was possible to accurately determine the degree of photodegradation, also in regions with a low fluorescence intensity. This aspect of 
our experimental set-up is of particular importance when the specimens contain structures with widely varying concentrations of the molecules identified by the fluorescent labels, so that large fluorescence intensity differences exist between structures labelled with different fluorophores.

A disadvantage in methods for multiple fluorescent labelling studies using single wavelength excitation and fluorophore identification based on emission spectrum characteristics is that, in order to prevent cross-talk between the fluorophores, only a small part of the emission spectrum, depending on the fluorophores, can be used for detection. The signal-to-noise ratio in the images obtained in this way is determined by the extent of overlap of the emission spectra of the fluorophores. In our method, the fluorophores should have similar excitation spectra, but no restriction is placed on the emission spectra of the fluorophores. The entire emission spectrum of all fluorophores is detected simultaneously. This leads to a potentially higher signal-to-noise ratio.

A possible drawback of our method is that it relies on the comparison of the intensities in two images, separated in time. This requires a good mechanical stability of the experimental set-up on time scales relevant to the experiment. Fortunately, most quality microscope stands, provided that they are placed in a stable environment, exhibit only small amounts of mechanical drift, so that such effects should not impose practical limitations on the method.

A particularly elegant feature of our approach to double fluorescent labelling studies is that when the degree of photodegradation is dependent on the detailed energy level structure of the fluorophores, the present method can be used to study the microenvironment of the fluorophores. Differences in the local environment result in variations of the observed degree of photodegradation with some fluorophores. The underlying cause of these variations is not the subject of this paper, but it is clear that a photodegradation analysis along the indicated lines can be useful for the study of the photodynamics of fluorophores in relation to their environment. Alternatively, if the relationship between the rate of photodegradation and the environmental effects are known, such data can be used for characterizing the microstructure of the specimen. The variation of the experimentally determined degree of photodegradation depends not only on differences in the microenvironment of the fluorophores, but also on variations of the excitation intensity over the field of view. Firstorder estimates of the relative magnitude of the excitation intensity variations indicated that these were at least an order of magnitude smaller than the variation of the degree of photodegradation reported here (results not shown). This means that, for these specific specimens, the variation of the degree of photodegradation is dominated by differences in the microenvironment.
Summarizing, we can say that structure-orientated photobleaching analysis can either be used to as an effective and simple tool for image decomposition in double fluorescently labelled specimens or, alternatively, for the determination of local variations of photodegradation and the underlying environmental factors.

\section{Acknowledgments}

We would like to thank Ms K. van der Kraan for preparing all specimens used in this study. This research was supported by the Stichting Technische Wetenschappen (STW), Utrecht, The Netherlands, under grant no. ANS 33.2941.

\section{References}

Bracewell, R. (1965) The Fourier Transform and its Applications. McGraw-Hill Book Company, New York.

Brakenhoff, G.J. \& Visscher, K. (1992) Confocal imaging with bilateral scanning and array detectors. J. Microsc. 165, 139146.

Brakenhoff, G.J. \& Visscher, K. (1993) Imaging modes for bilateral confocal scanning microscopy. J. Microsc. 171, 17-26.

Brakenhoff, G.J., Visscher, K. \& Gijsbers, G. (1994) Fluorescence bleach rate imaging. J. Microsc. 175, 154-161.

Carlsson, K., Aslund, N., Mossberg, K. \& Philip, J. (1994) Simultaneous confocal recording of multiple fluorescent labels with improved channel separation. J. Microsc. 176, 287-299.

Carlsson, K. \& Mossberg, K. (1992) Reduction of cross-talk between fluorescent labels in scanning laser microscopy. J. Microsc. 167, 23-37.

Carmo-Fonseca, M., Ferreira, J. \& Lamond, A.I. (1993) Assembly of snRNP-containing coiled bodies is regulated in interphase and mitosis - evidence that the coiled body is a kinetic nuclear structure. J. Cell Biol. 120, 841-852.

DeBasio, R., Bright, G.R., Ernst, L.A., Waggoner, A.S. \& Taylor, D.L. (1987) Five-parameter fluorescence imaging: wound healing of living Swiss 3T3 cells. J. Cell Biol. 105, 1613-1622.

Grande, M., Van der Kraan, I., Van Steensel, B., Schul, W., De The, H., Van der Voort, H.T.M., De Jong, L. \& Van Driel, R. (1996) PML-containing nuclear bodies: their spatial distribution in relation to other nuclear components. J. Cell. Biochem. 63, 280-291.

Larkin, K.G., Fairman, P.S., Farrant, D.I. \& Oreb, B.F. (1991) Restoration of shadow details in projected fringe profilometer images. DICTA-91, Conference on Digital Image Computing: Techniques and Applications, pp. 237-243. Australian Pattern Recognition Society, Monash University, Clayton, Victoria, Australia.

Loken, M.R., Parks, D.R. \& Herzenberg, L.A. (1977) Two-color immunofluorescence using a fluorescence-activated cell sorter. J. Histochem. Cytochem. 25, 899-907.

Pawley, J.B. (1995) Handbook of Biological Confocal Microscopy. Plenum Press, New York.

Press, W.H., Teukolski, S.A., Vetterling, W.T. \& Flannery, B.P. (1992) Numerical Recipes in C. Cambridge University Press, Cambridge. 
Schul, W., Groenhout, B., Koberna, K., Takagaki, Y., Jenny, A., Manders, E.M.M., Raska, I., Van Driel, R. \& De Jong, L. (1996) The RNA $3^{\prime}$ cleavage factors CstF-64kD and CPSF-100kD are concentrated in nuclear domains closely associated with coiled bodies and newly synthesized RNA. EMBO J. 15, 2883-2892.

Stuurman, N., De Graaf, A., Floore, A., Josso, A., Humbel, B.,
De Jong, L. \& Van Driel, R. (1992) A monoclonal antibody recognizing nuclear matrix-associated nuclear bodies. J. Cell Sci. 101, 773-784.

Ten Kate, T.K., Van Balen, R., Smeulders, A.W.M., Groen, F.C.A. \& Den Boer, G.A. (1990) SCILIAM, a multi-level interactive image processing environment. Pattern Recognition Lett. 11, 429-441. 